\title{
The Hierarchy Myopia of Organizational Learning*
}

\author{
NAMGYOO K. PARK ${ }^{* *}$ \\ Seoul National University \\ Seoul Korea \\ KIRA CHOI*** \\ London Business School \\ United Kingdom \\ JINJU LEE \\ Seoul National University \\ Seoul, Korea
}

\begin{abstract}
Previous studies have been interested in how to maximize both the efficiency and the effectiveness of organizational learning. On the flipside, some studies have investigated the critical barriers to learning. We suggest organizational hierarchy as another cause and theoretically explore how it can deter learning performance. Specifically, we argue that the configuration of structure determines a prevalent form of learning method in an organization to consequently affect its learning performance. Using simulation modeling, we show that non-hierarchical organizations may be a better learning environment than hierarchical organizations. We also show that the contextual factors, such as problem complexity and member regrouping, may affect the base-line result. This study subsequently calls for further attention be paid to the key issues concerning the hierarchy and organization learning performance.
\end{abstract}

Keywords: myopia of learning, hierarchy, organizational learning performance

* This study was supported by the Institute of Management Research and the Center for Global Business and Research at Seoul National University

** Graduate School of Business, Seoul National University, email: npark@snu.ac.kr

*** Corresponding author, London Business School, email: kchoi@london.edu

**** Graduate School of Business, Seoul National University, email: j.jinju.lee@gmail. com 


\section{INTRODUCTION}

The myopia of learning, a critical constraint to organizational learning, has been the focus of active academic investigation over the last few decades (Crossan, Lane and White 1999). Previous studies have defined the myopia of learning as an organization's inability to maximize the scope and depth of knowledge search, thereby hampering its overall effectiveness and learning efficiency (e.g., Levinthal and March 1993). Several types of myopic bias have been identified in terms of time, location, and previous experiences, typically depicting a firm's propensity to focus more on shortterm outcomes rather than long-term outcomes, to favor local search rather than distant search, and to oversample successes and undersample failures (Hayward 2002; Rosenkopf and Almeida 2003).

Hierarchy refers to the intra-organizational structure in which individuals are arranged in a cascade of authority and communication relations (March 1994). A key structure that sustains most organizations, hierarchy may greatly influence both the efficiency and the effectiveness of organizational learning. In an organization, hierarchy tends to specify the structural links and processes that determine the information to be circulated, the specific channels that distribute information, and the organizational members that are to be informed. It also defines an explicit span of control that affects the behavior of organizational members, such as external boundary spanning (e.g.,Williamson 1991), managerial cognition (e.g., Gavetti 2005), decision making (e.g., Mihm, Loch, Wilkinson and Huberman 2010), search (e.g., Rivkin and Siggelkow 2003) and information processing (e.g., Aoki 1986).

Previous studies have provided a reasonable foundation on a significant association between the hierarchical structure and organizational learning performance. For example, centralized decision making enabled by the hierarchy can stabilize search, reduce failure risk, and lead to faster decision making (e.g., Siggelkow and Levinthal 2005; Siggelkow and Rivkin 2005). Other studies have argued that the hierarchy can condense and distort the information flow, reduce organizational flexibility, inhibit unexpected discoveries, and increase time to find a quality solution (e.g., Burns and Stalker 1961; Jablin, Putnam, Roberts 
and Porter 1987). These studies leave a reasonable room for further investigation about the relationship between the hierarchy implemented and organizational learning. For example, Radner (1993) investigated how decentralization of information can result in the heterogeneous information-processing behaviors among different decision-makers in an organization. Although this line of work laid foundation for predicting how the level of hierarchy can influence various information-processing rule in a large organization, it has provided somewhat limited implications on organizational learning. Organizational learning can be more complex to strictly apply existing research on hierarchy and decision-making because; (1) organizational learning does not depend on certain rules whereas decision-making mainly depend on decision-making rules, (2) organizational learning is more interactive process than decisionmaking process, and (3) the outcome of organizational learning is harder to observe than decision-making. Therefore, we seek here to answer important but relatively unexplored research questions on which conditions a hierarchy may increase or decrease learning performance, and how firms with a typical hierarchical structure should configure their learning environment to improve learning performance. To investigate our research questions, we first draw on organizational learning literature in order to explain the characteristics and performance consequences of two distinct learning methods - horizontal and vertical learning - that are likely to emerge in the presence/absence of the hierarchy. We also draw on theoretical arguments regarding knowledge management, information system, and organizational communication in an attempt to predict the relationship between the hierarchical structure and learning performance under various conditions.

We provide three propositions related to the relationship between the hierarchical structure and organizational learning performance, and further illustrate them using simulation models. Through the examination of varying conditions at multiple levels, our study reveals that the hierarchy can be a new source of learning myopia. Specifically, we argue that non-hierarchical organizations with horizontal learning produce a better learning performance than hierarchical organizations with vertical learning. We then assert that the high level of interdependency and the low decomposability of complex problems may impose greater constraints to organizational learning, especially for hierarchical organizations. Lastly, we propose 
that regrouping project group members can be a possible solution for dealing with the typical learning disadvantages of hierarchical organizations. Indeed, identifying and overcoming the root cause of the myopia of learning should be a central concern in most organizations. This study subsequently calls for further attention be paid to issues concerning how hierarchical structure affects the organizational learning performance.

\section{LITERATURE REVIEW}

Over the past decades, scholars have extensively investigated various aspects of organizational learning. The first stream of research focused on how organizational learning is affected by the characteristics of knowledge itself, such as explicit vs. tacit (Nonaka 1994; Nonaka and Von Krogh 2009), declarative vs. procedural (Singley and Anderson 1989), and codified vs. noncodified knowledge (Zander and Kogut 1995; Zollo and Winter 2002). For example, Lawson and Lorenz (1999) argued that the outcome of organizational learning largely depends on tacit knowledge rather than explicit knowledge, and stressed the importance of the ability of individual members absorbing tacit knowledge. The second stream emphasized the mode of learning-such as scope, depth and speed-as a critical determinant of organizational learning (e.g., Carley 1992; March 1991). For example, March (1991) highlighted the balancing of exploitation and exploration as an efficient mode of learning for improved performance. The third stream examined the procedural aspects of organizational learning behavior, such as knowledge search (e.g., Knudsen and Levinthal 2007), knowledge creation (e.g., Gupta, Tesluk and Taylor 2007), knowledge transfer (e.g., Cohen and Levinthal 1990), and knowledge retention (e.g., Moorman and Miner 1997). Moorman and Miner (1997), for instance, examined the effect of organizational memory on organizational learning performance, while Majchrzak, Cooper and Neece (2004) investigated how organizations reuse retained knowledge. The fourth stream centered on how learning was affected by organizational characteristics such as structure (Bunderson and Boumgarden 2010; Fang, Lee and Schilling 2010), culture (Weber and Camerer 2003), identity (Kane, Argote and Levine 2005), and inter-firm alliances such as joint ventures (Hansen 2002). For 
instance, Fang, Lee and Schilling (2010) showed that semi-isolated subgroups within organizations, rather than isolated or fully connected ones, best promote learning. The last stream examined the question of how environmental conditions, such as industrial trait - whether it is high-tech or low-tech, level of uncertainty, and competition structure - affect organizational learning (e.g., Kim and Rhee 2009; Levitt and March 1988). For instance, a high degree of uncertainty about past successes tends to create significant stress for organizations, eventually decreasing the effectiveness of learning (Daft and Weick 1984).

\section{Myopia of Learning and Hierarchy}

While most previous studies have been interested in how to maximize both the efficiency and the effectiveness of organizational learning, some studies have investigated the critical barriers to learning, labeled as "the myopia of learning" (Levinthal and March 1993). The myopia of learning is recognized as the phenomenon where the overall effectiveness and the efficiency of learning can be hampered by the possibility that organizations may not maximize the scope and depth of knowledge-seeking behavior in terms of time, location, outcomes of previous actions, and so on.

The myopia of learning may occur due to a variety of factors, such as cognitive constraints, resource scarcity, the lack of learning capability, ineffective learning routines, and procedural inefficiency. Cognitive constraints, for example, may distort managerial perception and lead to suboptimal decision making (March and Olsen 1975). Resource scarcity could inherently build significant tension around resource allocation, eventually constraining the scope and depth of knowledge-seeking behavior. The lack of organizational learning capability, such as limited absorptive capacity (Cohen and Levinthal 1990), could delay the necessary procedures for a firm's survival, such as valuing, assimilating and applying new knowledge (Szulanski 1996). Even when dealing with the same learning task, organizational learning processes may allow different organizations with almost equivalent experience levels to exhibit different levels of performance improvement (Pisano, Bohmer and Edmondson 2001). Procedural inefficiency often inhibits knowledge transfer across teams, functions, and geographical locations (Darr and Kurtzberg 2000; Gittelman 2007). Most 
organizations commonly face the aforementioned challenges in their learning processes. Thus, identifying and overcoming the causes of the myopia of learning is undoubtedly a central concern for most organizations.

Hierarchy, the most pervasive feature across almost all organizations, is the intra-organizational structure in which individuals are arranged in a cascade of authority, status, and communication relations (March 1994). As the backbone of most organizations, it has a significant influence on cognition, resource availability, capability building, decision making, and internal interactions. Hierarchy also determines the contents of learning and the specific channels that are designed to access both internal and external knowledge since it specifies the structural links, systems and processes, and groupings within organizations (Rivkin and Siggelkow 2003). It is also an explicit span of control that affects the scope of organizational boundary spanning (Williamson 1991). For example, senior managers at the upper levels of the hierarchy often allocate tasks and delegate decisions, provide incentives, and structure intra-organizational communication. While most organizations seek to achieve the best combination of speed, quality, and minimal failure with the help of the hierarchical structure, they cannot avoid the possibility of the hierarchy becoming another cause of the learning myopia. In fact, some studies have recognized that hierarchy hampers efficient learning processes by distorting or condensing information flow in the chain of sequential commands (Jablin et al. 1987; Senge 1990). Hierarchy also restricts the flow of information, which may, in turn, cause firms to overlook opportunities for innovation and discovery (Burns and Stalker 1961; Williamson 1991).

Previous studies have linked hierarchy with many other theoretical constructs such as decision making (e.g., Mihm et al. 2010), search (e.g., Rivkin and Siggelkow 2003), and information processing (e.g., Aoki 1986). Taken together, these studies provide a reasonable foundation on a significant association between the hierarchical structure and organizational learning performance. For example, centralized decision making enabled by the hierarchy can stabilize search, reduce failure risk, and lead to faster decision making (Siggelkow and Rivkin 2005). Meanwhile, the hierarchy can condense and distort the information flow, reduce organizational flexibility, inhibit unexpected discoveries, and increase time to find a quality 
solution (e.g., Burns and Stalker 1961; Jablin et al. 1987). However, these studies remain rather inconclusive, and leave reasonable room for further sophistication about the relationship between the hierarchy implemented and organizational learning. Therefore, we define the concept of hierarchy myopia as the phenomenon in which a hierarchy decreases the efficiency and the effectiveness of organizational learning by influencing cognition, information flow, decision making, boundary spanning, and resource availability among others. We endeavor to expand the literature on learning myopia by investigating how the hierarchy affects organizational learning performance, and how such result varies under different contexts.

To formulate our propositions, we first assumed that the typical learning method used in hierarchical organizations are significantly different from that used in non-hierarchical organizations. According to Edmondson, Bohmer and Pisano (2001), the hierarchical structure can determine the learning method of organizations in several ways. Leaders at the upper levels of the hierarchy often coordinate and assign tasks to group members, decide what the necessary patterns of internal interactions will be, and influence group members' views; through this, they develop the most efficient and effective learning methods. The typical interaction patterns within organizations with multiple hierarchies can be strongly contrasted with those within organizations that lack hierarchy. Previous studies noted that in organizations with a hierarchy, learning most likely occurs through vertical interactions from top to bottom, whereas in organizations without hierarchy, learning occurs through horizontal interactions between multiple organizational members (Nickerson and Zenger 2004; Nonaka 1994). Thus, we define vertical learning as a form of learning typically observed in hierarchical organizations where members obtain new knowledge from those above and below their respective hierarchical levels uni-directionally, and horizontal learning as a form of learning that usually prevails in non-hierarchical organizations, where participants acquire new knowledge from colleagues regardless of their position multidirectionally. To investigate how hierarchy triggers the learning myopia in organizations, we compare the learning performances of organizations with two contrasting structures employing two distinct learning methods, vertical learning in hierarchical organizations and horizontal learning in in non-hierarchical organizations. 


\section{PROPOSITION}

Assuming that all others are equal, an organization's learning method can significantly influence the two critical determinants of organizational learning performance, the contents and the process of learning (Fiol and Lyles 1985; Huber 1991). First, let us consider why and how the contents of learning differ between the organizations with non-hierarchical and hierarchical structures. In the case of non-hierarchical organizations, where horizontal learning is highly likely to take place, multiple actors have access to different sets of information and flexibly which enable them to engage in various activities without any pre-determined chain of command (Carley 1992). Such an autonomous atmosphere tends to broaden the scope of the knowledge sets of subordinates to be more heterogeneous. Vertical learning, which is highly likely to be typical in most hierarchical organizations, is characterized by a ranking system consisting of a leader and multiple subordinates. The role of a leader is often to understand and utilize the existing knowledge base and structure, and such a role is usually assigned to the person who is believed to possess the best available knowledge set (Carley 1992). Thus, the primary tasks of a leader are to design possible interaction patterns among members, to structure the sublearning processes for subordinates, and to designate what to learn and from whom to learn (Baldwin and Clark 2000; Nickerson and Zenger 2004). Such a heavy dependence on the leader and pre-set routines for organizational learning may often restrict the contents of learning and the scope of knowledge (Perretti and Negro 2006). Thus, compared to non-hierarchical organizations, hierarchical organizations may subsequently decrease the utility of learning by narrowing the range/breadth of knowledge sets, and homogenizing knowledge sets.

The two learning methods may lead to distinctly different learning processes. The efficiency and effectiveness of a learning process depends on how internal communication is carried out, how quickly knowledge is shared among members, and how specific knowledge can be integrated with other knowledge (Kogut and Zander 1992; Nonaka 1994). Hierarchical organizations usually rely on communication across hierarchies, such as vertical reporting systems or order routines, and knowledge flow subsequently occurs 
along vertical communication channels (Mueller 1994). Such typical communication behavior often deprives group members of discretion in dealing with new problems, restricts knowledge flows with multiple red tapes, and reduces their motivation to share knowledge with other members, subsequently decreasing knowledge sharing activities within organizations. The aforementioned characteristics of the hierarchical organizations may hinder the securement of complementary knowledge in a timely manner; this further limits the voluntary integration of knowledge across multiple hierarchical layers. Group members in non-hierarchical contexts, on the other hand, can freely interact with others, as all members are of equal status. Such equal status may make members more likely to share knowledge and increase members' motivation to integrate their specialized knowledge sets (Nickerson and Zenger 2004). Non-hierarchical organizations usually foster an environment where members actively interact and extensively participate in open communications (Galbraith 1974). Furthermore, the greater the diversity of individuals involved, the wider the scope of knowledge being integrated and the greater the complementarity of each knowledge set (Grant 1996). Thus, compared to hierarchical organizations, non-hierarchical organizations may promote the circulation of better ideas and their integration (Kogut and Zander 1992). Due to the above differences in both the content and the process of learning, we propose that:

Proposition 1: Other things being equal, the organizational learning performance of horizontal learning will be superior to that of vertical learning.

Previous studies acknowledged that problem complexity may significantly undermine learning performance (Kauffman 1995). Organizations, especially in high-tech industries or knowledgeintensive industries, often encounter complex problems. A complex problem is conventionally understood as a problem whose subcomponents are highly interdependent (Nickerson and Zenger 2004). The typical resolution of a complex problem requires: 1 ) heterogeneous inputs from various backgrounds and experiences and 2) the sophisticated management of highly interdependent subcomponents of knowledge (Kauffman 1995; Simon and Simon 1962). Hence, complex problems usually require the participation 
of multiple actors possessing distinctly different types of knowledge and active interactions among those participants (Foss 2007; Nickerson and Zenger 2004). By doing so, participants can identify relevant knowledge sets and multiple combination sets, which can maximize the probability of discovering a valuable solution.

All other things being equal, problem complexity can significantly moderate the relationship between hierarchical structure and learning performance. Complex problems may affect various antecedents of organizational learning performance, such as learning capability, available knowledge, learning contexts, and learning methods. In non-hierarchical organizations, diverse knowledge sets help members interpret ambiguous and complex issues, by promoting the discussion and debate of competing perspectives and approaches to identify an optimal solution and to increase their assessment accuracy of the situation (Daft and Lengel 1986; Jansen, Van Den Bosch and Volberda 2005). The presence of such knowledge stimulates multiple trials of cross-fertilization or (re-) combinations of different kinds of knowledge, which is only possible through extensive lateral interactions that facilitate the sharing of specialized knowledge and the development of common cognitive maps (Nickerson and Zenger 2004). For instance, IDEO, a renowned industrial product design firm, maximizes the power of diversity for their creative new product development projects. It assigns professionals with various backgrounds of at least 40 industries to a project group. Project groups often lack pre-designated leaders, and most members do not have titles. Extensive interactions, frequently observed in non-hierarchical organizations than hierarchical organizations, may promote learning from trial and error, allowing fast evaluation of multiple alternatives (Foss 2007). Members can also make the acquisition of critical knowledge possible by quickly finding and sharing complementary knowledge (Coleman 1988; Hansen 1999). Thus, we propose that:

Proposition 2: Other things being equal, the gap between the organizational learning performance of horizontal learning and vertical learning will be greater when problem complexity is high.

To develop the previous propositions, we relied on the fundamental assumption that the composition of learning groups would remain consistent. However, in the real business world, member regrouping 
occurs frequently within an organization. Consider the typical context of an R\&D division in large high-tech organizations, where a few hundred engineers collaborate to develop new technologies or products. All operating project groups are composed of multiple members. When a project is completed, members are frequently rearranged across group boundaries to initiate another task that may be or may not be related to their previous tasks. In particular, many firms intentionally implement member changes to achieve their objectives and to quickly adapt to sudden environmental changes (Brady and Davies 2004). Thus, we would like to extend our assumption and integrate possible member regroupings in our argument. Following the concept of the "open group," (Choi and Thompson 2005), we define member regrouping as "member exchange across different groups within the same organization" where a work group is disassembled and reassembled with members regardless of their affiliated groups.

Member regrouping can significantly affect an organization's learning performance both negatively and positively since it exerts a substantial influence on important components of the learning environment such as structure and process (Arrow and McGrath 1995; Choi and Thompson 2005). Earlier studies that examined the detrimental effects of member turnover on performance highlighted that members may take time to adjust themselves to a new learning context (Naylor and Briggs 1965), disrupt shared knowledge or mental models among existing members (Cannon-Bowers and Salas 2001), and subsequently tend to interfere with existing routines for learning (Moreland and Levine 1982). However, recent studies have elucidated the positive aspects of member turnover such as fostering the need for creativity and exploration of new knowledge (e.g., Choi and Thompson 2005; Gruenfeld, Martorana and Fan 2000). These studies emphasized that new members can add entirely different or explorative knowledge to existing knowledge bases, possibly replace redundant or inefficient knowledge, establish new learning routines, and improve existing learning processes.

When member regrouping is introduced to the on-going process of organizational learning, hierarchical organizations can exploit more benefits of member regrouping than non-hierarchical organizations. In hierarchical organizations, newly grouped members may bring fresh and diverse knowledge into their project groups that would otherwise consist of rather homogeneous knowledge sets (Levine 
and Moreland 1994). Such heterogeneous knowledge will facilitate knowledge diffusion and sharing across an entire organization since member regrouping provides new opportunities to work with new members (Choi and Thompson 2005). These changes can help organizations using the vertical learning method to overcome their fundamental weaknesses, the inherent homogeneity of knowledge sets within the project groups and the relatively low degree of knowledge sharing across project groups. On the other hand, the possible downsides of member regrouping are likely to be less severe for non-hierarchical organizations than for hierarchical organizations. When member regrouping occurs, the first priority that project groups have to deal with is the reestablishment of learning routines, collaboration procedures, communication channels, and so on. In hierarchical organizations, however, project groups can quickly deal with most of these issues since they can rely on existing spans of control, formal rules and organizing principles (Grant 1996). A relatively high degree of formalization of collaboration may reduce the necessary time and resources for these set-up procedures (Pentland and Rueter 1994). Therefore, when member regrouping occurs, hierarchical organizations are likely to exploit more benefits and experience less detrimental effects than non-hierarchical organizations. Thus, we propose that:

Proposition 3: Other things being equal, member regrouping across project groups will increase the organizational learning performance of vertical learning more than that of horizontal learning.

\section{MODEL}

To illustrate our propositions on how hierarchy affects organizational learning performance, we devise a simulation model of an organization where individuals learn by interacting with others mainly within the boundary of project groups. We construct a simulation model, similar to the agent-based model presented by March (1991), and Miller, Zhao and Calantone (2006). In March (1991)'s seminal work, a simulation model reflects reciprocal learning within an organization. Through a socialization process, individuals learn from an organizational code that is composed of shared 
language, beliefs, and practices, and the organizational code, in turn, refines itself based on the best performing individuals. This iterative interaction helps individuals to self-reflect on existing ideas and adopt better and new ones (Fang, Lee and Schilling 2010). Miller, Zhao and Calantone (2006)'s model extended March (1991)'s model of mutual learning between an organization and its members by adding interpersonal learning. Following above studies, we view individuals as carriers of ideas and knowledge, and organizational learning as a property that emerges from interactions among individuals in an organization. Individuals interact with others, who may influence them to adopt new ideas and to discard old ideas. Extending from previous works, we develop an organizational learning model wherein the learning of organizational members are affected by two different learning methods which are typically observed in hierarchical or non-hierarchical organizations. We also consider task complexity, member regrouping across groups, and learning rate to qualify our findings. Our simulation model has four main entities - an external reality, individuals, groups and organization.

\section{External Reality}

Following March (1991), there exists the reality the organization seeks to learn about. We describe reality as having $\mathrm{m}$ dimensions, each of which has a value of either 1 or -1 . The probability that any one dimension will have a value of 1 (or -1 ) is 0.5 ; values are randomly assigned.

\section{Individuals}

There are $\mathrm{n}$ individuals in the organization. Each of them holds $\mathrm{m}$ dimensions of knowledge sets or ideas that may or may not correspond to the dimensions of the reality. Each of the belief also has a value of 1 or -1 , again, randomly assigned.

\section{Groups}

Individuals are assigned to groups which designate the learning boundary for individuals belonging to the group. Each group, composed of multiple individuals, is assigned with different projects 
about which individuals learn new knowledge through interactions with co-workers in the same group. To control for the potential effect of interactions between groups, our model does not include possible interactions beyond the boundary of one's group. We construct an organization to be comprised of $\mathrm{x}$ groups wherein each group is comprised of multiple individuals.

\section{Organization}

An organization is comprised of $\mathrm{n}$ individuals and $\mathrm{x}$ groups. Our model is different from the March (1991) in that an organization is seen as a complex system wherein learning can occur from direct interaction with leader or group members, depending on the learning method of an organization.

\section{Learning}

When individuals first join a project group, they possess a heterogeneous set of beliefs or knowledge sets. At each learning period, individuals interact with, and compare their own knowledge sets to those of other project group members. Once it has been determined that the knowledge sets of others match the reality better than theirs, individuals update their own knowledge sets. Following March (1991), we view these processes of sharing, comparing, interpreting, and updating beliefs, as organizational learning.

The organization reaches long-run equilibrium performance when its performance no longer improves. As superior ideas are diffused, there comes a point when the entire organization converges upon a set of beliefs and there are no more superior-performing individuals to learn from. The learning performance of an individual is measured as the sum of each individual's number of dimensions that correctly matches the given external reality at every time period. The learning performance of an organization is measured as the average learning performance of all of the individuals in the organization.

There is an inherent uncertainty in the individual's ability to evaluate whether certain sets of beliefs adhere to reality or not. To address this, we adopt March (1991)'s decision rule of learning, $\mathrm{p}$, which is the probability of individuals deciding to learn from others once they judge that the knowledge set of others is higher- 
performing or dominant than theirs. In this paper, we fix $\mathrm{p}$ as 0.3 in our baseline model and give variations in the sensitivity analysis. This learning rate is commonly used in various learning models and it is based on the large body of research on social decision schemes and it has been supported by numerous studies of social decision making (Castore, Peterson and Goodrich 1971; Davis, Kerr, Atkin, Holt and Meek 1975).

\section{Two Contrasting Learning Methods}

To show how the presence and the absence of a hierarchical structure affect organizational learning performance, we consider somewhat extreme types of learning methods in an organization: (1) an organization that is governed by hierarchy, which typically exhibits vertical learning, and (2) a non-hierarchical organization which generally utilizes horizontal learning.

First, for vertical learning, the presence of hierarchy is represented by the two hierarchical layers in a group - a leader and subordinates. Based on the inter-personal learning model of Miller, Zhao and Calantone (2006), we modeled the learning to occur uni-directionally, from a designated leader to the rest of the group members. The leaders of each group are the highest-performing individuals in their groups, and they serve as the main source of knowledge. Group members update their knowledge with the superior knowledge sets of the designated leader.

Second, for horizontal learning, the absence of hierarchy is represented by the context where the source of knowledge will not be confined to certain individuals and the interactions among group members are bi-directional. In March (1991)'s learning model, individuals interact with and learn from an organization-specific knowledge, which simultaneously, learns from the best-performing individuals. Similarly, we build a learning model where group members learn from group-specific knowledge composed of the best available knowledge within the group, which simultaneously, learns from the group members.

Figure 1 illustrates the contrasting property of the two learning methods. Figure $1 \mathrm{~A}$ shows the most simplified structure of organizational hierarchy where only a single layer exists between the project group leader and group members while Figure 1B shows no layers among group members. 


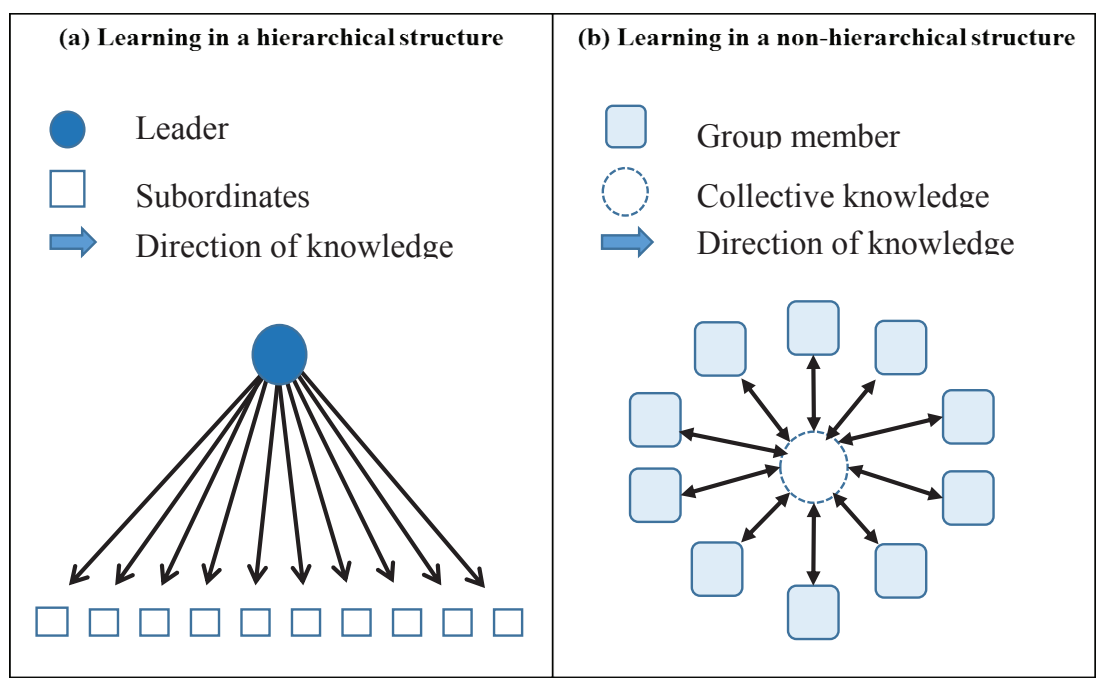

Figure 1. Organizational Structure and Learning Methods

\section{Problem Complexity}

To incorporate problem complexity, we construct a payoff function to show how the problem complexity conditions the calculation of learning performance. Following Fang, Lee and Schilling (2010), we adopt a generalized $\mathrm{m} / \mathrm{s}$ payoff function where $\mathrm{m}$ refers to the dimensionality of the problem and $s$ is a parameter through which we can change the interdependence of the problem. That is, the learning performance will not improve unless all of the interdependent dimensions jointly match the corresponding parts of reality. For the simplest case, when the problem complexity is very low (i.e., $s=1$ ), the dimensions are completely independent and the performance of each individual is calculated as the sum of the correct dimension score. However, increasing the value of $s$ makes a problem more interdependent. Consider a case when there are 10 dimensions of knowledge and all are grouped jointly ( $s=10, m=10$ ). If an individual holds less than 10 correct knowledge sets, then the payoff score will be 0 , while holding 10 correct knowledge sets will lead to a score of 10 . Thus, if $s=m$, the search problem is maximally interdependent; if any single element among the $m$ beliefs does not match the reality, the payoff for the whole set becomes zero. In our 
baseline model, we keep $s=1$ for a simple problem and $s=2,4,5$, $10,20,25$ for a complex problem with 100 dimensions $(m=100)$.

\section{Member Regrouping}

To incorporate member regrouping, our model reshuffles the members of all groups periodically. When member regrouping occurs, the existing groups are disassembled and randomly reassembled from the pool of individuals within an organization. To examine the significance of member regrouping, rather than regrouping a fraction of the individuals, we model all individuals to be subject to member regrouping. Regrouping all members in an organization allows us to observe the organizational learning performance in a context where learning routine and processes are completely dissolved and reestablished. To thoroughly explore the effect of member regrouping, we also parameterize different intervals of member regrouping. We compare cases where there is no member regrouping $(t=0)$, member regrouping occurs at every time period $(t$ $=1$ ), member regrouping occurs at every five time period $(t=5)$, and member regrouping occurs at every ten time period $(t=10)$.

\section{SIMULATION RESULTS}

We use the model described above to run a series of simulations that well-illustrate the impact of two different learning methods typical in hierarchical and non-hierarchical organizations. We first explore the impact of the learning method in a baseline model and then show the influence of problem complexity and member regrouping in an extended model. The simulation results reported here are based on the average of 200 iterations of the Monte Carlo simulation for each set of parameters until 100 time period.

\section{Baseline Model: Hierarchical Organizations vs. Non-hierarchical Organizations}

In the baseline model, we compare the organizational learning performance of two contrasting learning methods in a simple problem setting. To do so, we fixated the dimension of knowledge sets at $m=100$, the interdependency among the knowledge sets at $s$ 


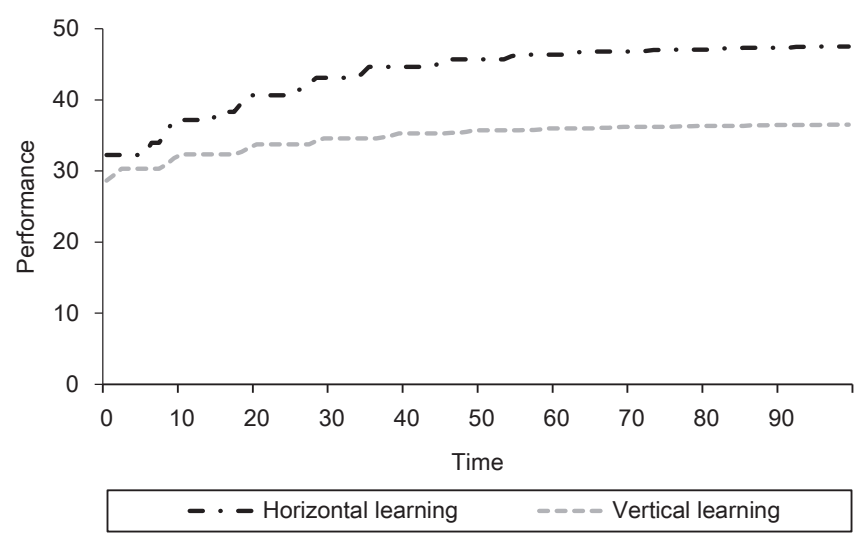

Figure 2. Organizational Learning Performance in a Simple Problem Setting

$=1$, learning rate at $p=0.3$, the number of individuals $n=100$ and the number of groups at $=10$.

Consistent with our proposition 1, Figure 2 shows that the organizational learning performance of non-hierarchical organizations is higher than that of hierarchical organizations. Although the initial score for both cases of learning start off somewhat similarly, the later-unfolded score exhibits a larger disparity; the long-term organizational learning performance of hierarchical organizations merely reached about two-thirds of the scores achieved by nonhierarchical organizations. This pattern of disparity is sustained throughout the simulation runs. The result is in line with our conjecture that the performance gap is driven by the differences in the contents and the processes of the two learning methods.

To better understand why such a critical discrepancy between the two forms of organizations exists, we further investigated how, depending on the form, learning performance varies among groups within each organization. Rather than simply aggregating and averaging the learning performance of all 10 groups, we calculated the variance among each group's learning performance. Figure 3 shows the simulation result on the variance of learning performance among groups in both hierarchical and non-hierarchical organizations, while keeping all the parameters consistent with the baseline model. The magnitude of performance variance is much larger for hierarchical organizations than for non-hierarchical 


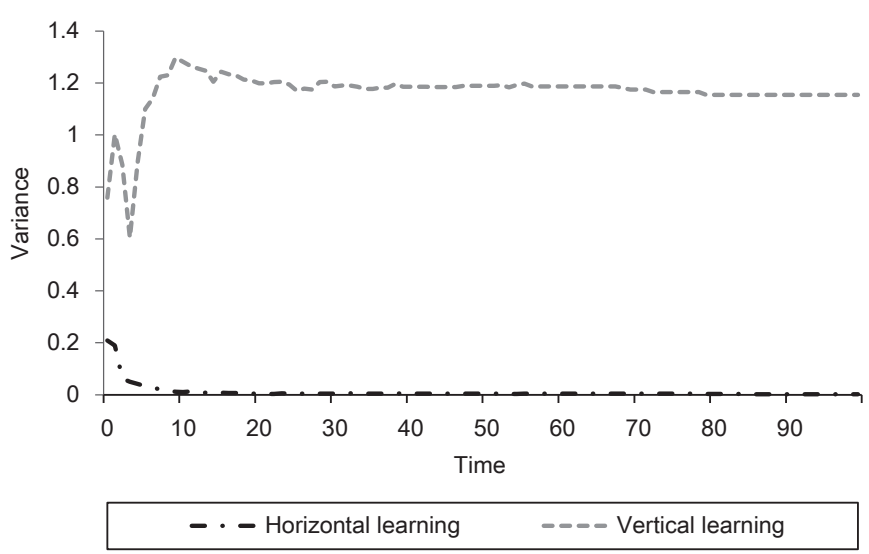

Figure 3. Performance Variance in a Simple Problem Setting

organizations. At the initial time period, the performance variance among groups of the hierarchical organizations fluctuates significantly. Over time, the variance gradually stabilizes as the organizational performance reaches equilibrium. Meanwhile, the performance variance among groups of non-hierarchical organizations shows a certain degree of variance at first, but immediately converges to no variance at all. Comparing the two results, the variance of the hierarchical organizations displays a much broader range than that of the non-hierarchical organizations, and the observed disparity persists throughout the time period. Such a persistent gap between the variance of the organizations may suggest that the learning performance of hierarchical organizations is highly bounded to or vulnerable to the capability of the leaders.

\section{Extended Model 1: The Role of Problem Complexity}

To illustrate the effect of problem complexity on learning performance, we vary the parameters $m(m=10,20,50,100,150)$ and $s(2 \leq s \leq 25)$ jointly. We keep all other parameters consistent with the baseline model. Figure 4 shows the learning performance of the two contrasting forms of organizations in a complex problem setting when $m=100$. We find that although the performance scores of both hierarchical and non-hierarchical organizations decrease, the performance gap between the two increases as the problem becomes more complex. When problem complexity is moderate $(2 \leq s \leq 10)$, 
the performance score of non-hierarchical organizations ranges between 80 and 100. However, hierarchical organizations show a lower range between 0 and 40 . When the problem complexity is extremely high with substantial interdependence among knowledge sets $(s \geq 20)$, the performance of hierarchical organizations hovers around 0 . The near-zero performance of hierarchical organizations for very complex problems suggests that hierarchical organizations are more susceptible to problem complexity than non-hierarchical organizations. When $s \geq 20$, the performance of the hierarchical organizations still manages to achieve an equilibrium performance score of 70 or above. Overall, both organizations suffer from problem complexity, while the degree to which they are affected differs.

We find that changing $m$ does not greatly affect the pattern discussed above. The superiority of non-hierarchical organizations is robust to changes in $\mathrm{m}$, regardless of the interdependence level of search problems (i.e., $s=2,4,5,10,20,25)$. An increase in $\mathrm{m}$ seems to only make the performance gap between hierarchical and non-hierarchical organizations more pronounced. Following preivous studies, we also checked whether the group size changes our findings. To examine the effect of group size, we specify a parameter $\beta$ that corresponds to the size of each group. We then set the parameter at either $\beta=5$ (a total number of groups as 20) or 10 (a total number of groups as 10). As shown in Figure 5, the variation of group size does not change our main findings in our baseline model. However, we find that performance score according to the size of the group is contingent upon the problem complexity. When the problem is comparatively simple (e.g., $s=1,2$ ), the performance scores are not sensitive to the size of the group. However, when the problem is rather complex (e.g., $s \geq 5$ ), larger groups $(\beta=10)$ clearly outperform smaller groups $(\beta=5)$ for both learning methods. Regardless, the magnitude of impact is greater for non-hierarchical organizations. Hence, our simulation result implies that organizations dealing with highly complex knowledge will benefit more from larger groups in the case of non-hierarchical organizations than in the case of hierarchical organizations.

\section{Extended Model 2: The Role of Member Regrouping}

In the previous section, we assumed that there would be no change in the members of the group. As explained above, we include 

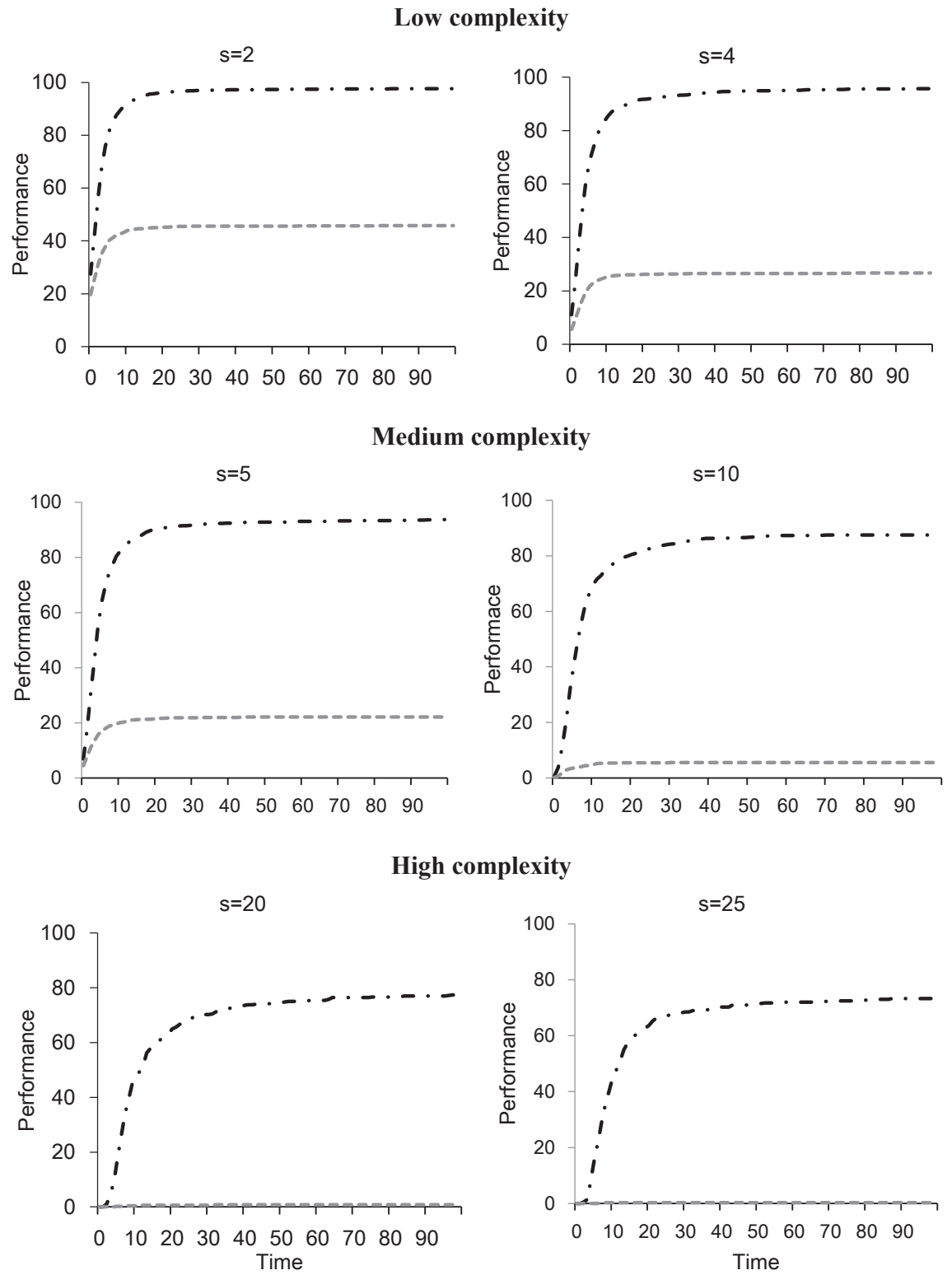

- - Horizontal learning

---- Vertical learning

Figure 4. Organizational Learning Performance in a Complex Problem Setting 


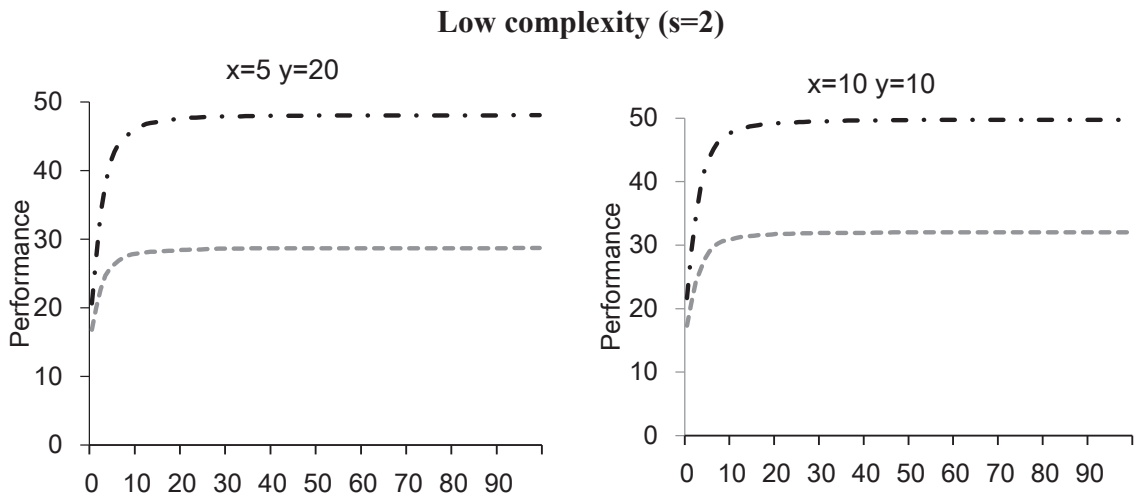

Medium complexity $(s=10)$
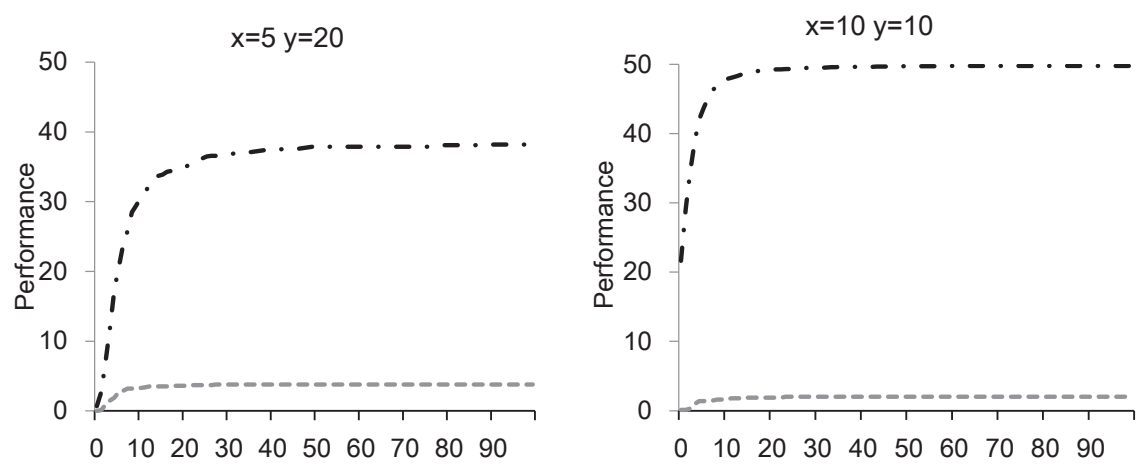

High complexity ( $s=25)$
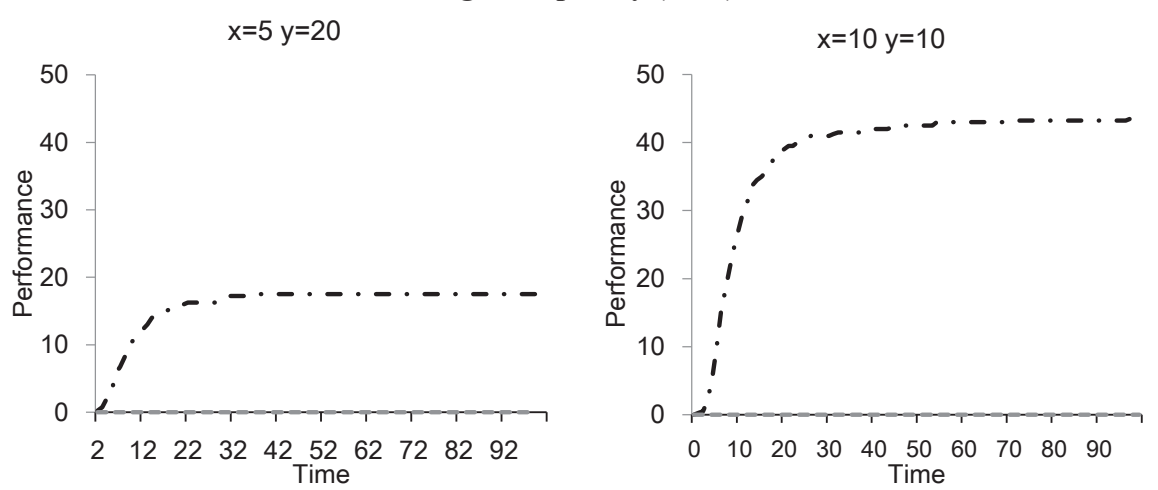

Figure 5. Effect of the size of subgroup 
the event of member regrouping in our simulation model while keeping other parameters the same as before $(m=50, n=100, x$ $=10, p=0.3)$. We designate member regrouping to take place by disassembling and randomly reestablishing all groups within the exiting boundary of an organization at a certain time period. To illustrate the effect of member regrouping, we compare the case where there is no member regrouping $(t=0)$, member regrouping occurs at every time period $(t=1)$, member regrouping occurs at every five time period $(t=5)$, and member regrouping occurs at every ten time period $(t=10)$. Figure 6 shows all simulation results with periodic member regrouping for both simple $(s=1)$ and complex $(s=5)$ problem settings. As shown earlier, when there is no member regrouping, non-hierarchical organizations outperform hierarchical organizations for both simple and complex problem settings. However, regardless of its interval (i.e., $t=10$ ), when member regrouping occurs, the learning performance of hierarchical organizations catches up with that of non-hierarchical organizations. This positive impact of member regrouping for hierarchical organizations becomes more intense with shorter intervals of member regroupings (i.e., $t=1$ or 5 ). The positive impact is so substantial that the learning performance of both organizations converge. In addition, non-hierarchical organizations also benefit from member regrouping since it clearly reduces the time necessary to reach the maximum learning performance score. However, compared to hierarchical organizations, the positive impact on the learning performance of non-hierarchical organizations is marginal. Overall, we find that member regrouping is consistently beneficial for organizations, especially for hierarchical organizations.

We conducted a few more sensitivity analyses to check the robustness of our findings. First, we vary the size of an organization ( $n=50,200$ and 400) and find that there is no substantive change in our results. Second, we vary the individual learning rate $(0.1 \leq p \leq$ 1) to see how our findings are affected in our baseline and extended models. Whether the learning rate is sufficiently small or big, these different learning rates do not change our reported simulation results, with the learning rate $p$ at 0.3 . Regardless of the learning rates and problem complexity, non-hierarchical organizations exhibits higher scores than hierarchical organizations. 

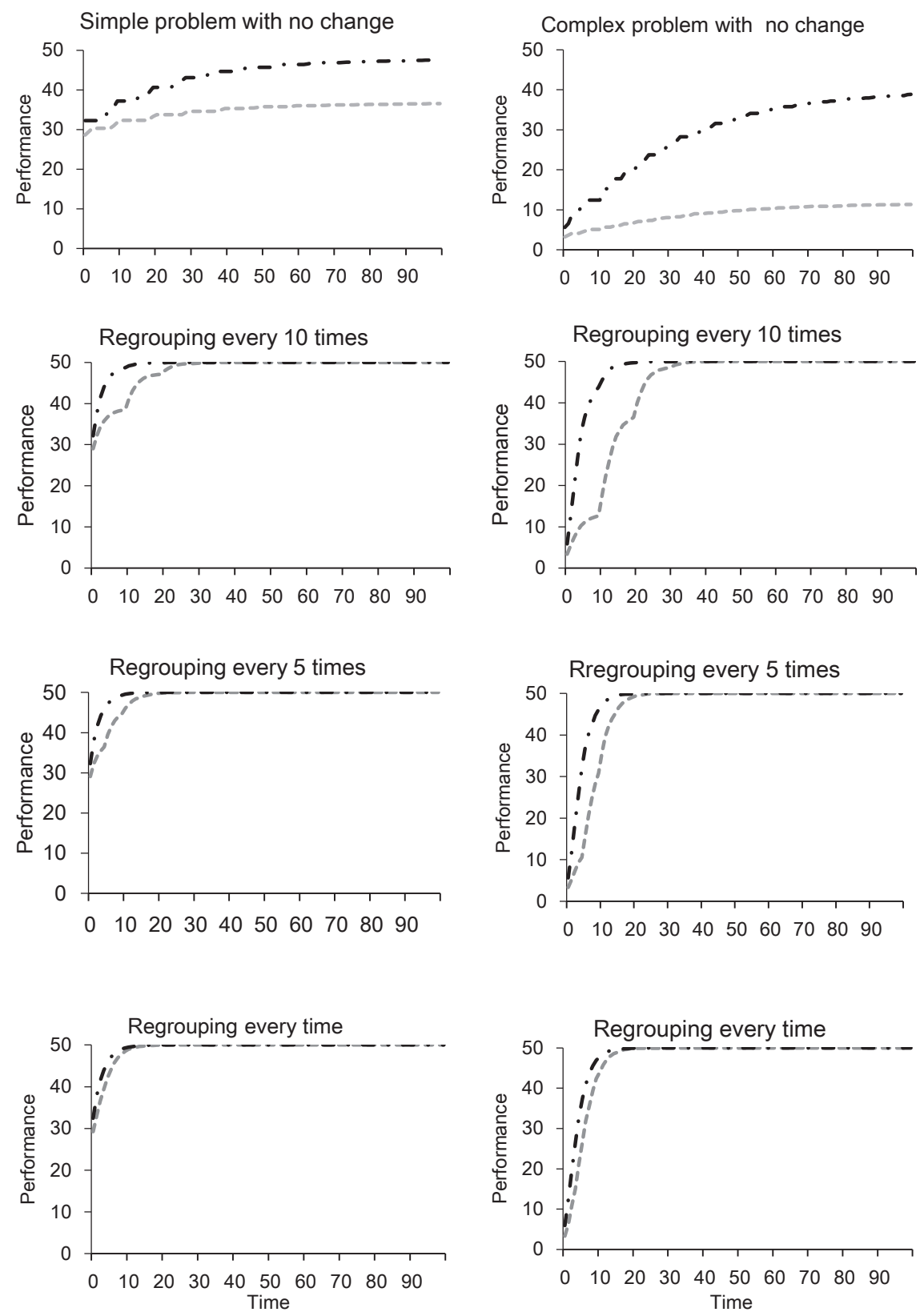

Figure 6. Effect of Different of Member Regrouping in Simple/Complex Problem Setting 


\section{DISCUSSION AND CONCLUSION}

In this study, we used a series of simulations to examine the relationship between hierarchical structure and organizational learning. Our study assumed that the configuration of hierarchical structure in an organization - whether an organization is arranged in a hierarchical or non-hierarchical manner - predetermines the learning method that governs the contents and process of learning, possibly bringing about significantly different organizational learning outcomes. We also suggested that contextual factors such as problem complexity and member regrouping may affect the learning performance of two different forms of organizations. The simulation result showed that a non-hierarchical organization is more effective than a hierarchical organization in terms of providing diverse learning contents, facilitating effective knowledge sharing, and enhancing problem solving capabilities. In addition, our results also provide implications on how different learning contexts can be applied to such outcomes. When firms are faced with different levels of knowledge complexity, the difference in the effectiveness of learning becomes more evident. Greater complexity, accompanied by higher interdependency, imposes more constraints to hierarchical organizations than non-hierarchical organizations, aggravating the learning performance in a much greater manner. These simulation results imply that hierarchical firms can be trapped in the hierarchy myopia of learning: a situation where a firm's inherent structure hinders its learning opportunity and process. However, our study also showed that hierarchical organizations can improve their learning performance by introducing an intentional measure. We showed that the configuration of member regrouping can help hierarchical organizations to overcome their inherent weaknesses and avoid the hierarchy myopia of learning.

Previous studies on organizational hierarchy typically viewed hierarchy as a means to enhance work efficiency and thus linked it with constructs such as decision making, search and information processing. However, in recent years, there have been efforts to investigate how hierarchy affects other learning-related constructs. For example, Nickerson and Zenger (2004) suggested that different forms of hierarchy -authority-based hierarchy vs. consensus-based hierarchy - play different roles in solving a complex problem. Our 
study provides additional insight into prior studies on learning myopia by pinpointing the mechanism through which hierarchy provides a myopic bias in learning. As noted previously, the myopia of learning occurs when an organization becomes short-sighted and fails to maximize its long-term learning benefits by engaging in more immediate, proximate and familiar learning activities. Suggesting a new notion of the hierarchy myopia, our study extends the learning literature by identifying the relationship between hierarchy and the myopia of learning, a phenomenon in which hierarchy may decrease the efficiency and effectiveness of organizational learning by influencing cognition, information flow, decision making procedures, boundary spanning, resource availability, and so on. In terms of the effect of hierarchy, we developed a conservative and parsimonious simulation model with only single layer of hierarchy. While most organizations tend to have more than a single level of hierarchy, the existence of multiple layers of hierarchy is likely to decrease the learning performance of vertical learning methods. Thus, our theoretical argument will also be relevant for mostly large organizations where managers often need to deal with multiple layers of hierarchy. However, the existence of multiple layers of hierarchy can be an unavoidable phenomenon for certain research contexts, such as investigating the bureaucratic aspects of organizational learning dynamics. Thus, future studies could clearly extend our research by integrating multi-level hierarchies and examine their differential effects on how collaboration should be sequenced, decisions should be delegated and the span of controls should be designed. For example, a few studies investigated hierarchical structure and search process, assuming that decisionmaking processes may occur through multiple chains of hierarchy (e.g., Carley 1992; Mihm et al. 2010). Our findings also imply that depending on the organizational structure, the quality and the type of knowledge production can differ. In particular, inimitability or substitutability of the knowledge produced from cooperative learning process is highly likely to be higher than leader-focused learning process, as designing a similar cooperative learning process will be more difficult than the other. Therefore, viewing different learning processes as a firm-specific capability and/or resource can further extend the implication of our findings.

We also add an important theoretical insight to the relationship between organizational hierarchy and learning performance by 
emphasizing different tendencies in the formation, circulation, and integration of knowledge. Starting from Arrow (1985) and Galbraith (1974)'s works, many studies have extensively investigated the roles and key features of knowledge and information flow within an organization. They identified horizontal and vertical information structure and explicitly compared their characteristics and efficiency in various dimensions, such as how effectively information is communicated, decisions are coordinated, or knowledge is shared (e.g., Aoki (1986); (Grant 1996; Schulz 2001; Tsai 2001). Previous studies found that the hierarchy-based information structure is much more effective in coordinating knowledge for multiunit organizations (Tsai 2001), and effective in facilitating the new knowledge flow that is acquired by low-level subunits to upperlevel subunits (Schulz 2001). However, only a few studies associated these with organizational learning performance. We explain the characteristics of and the performance consequences of learning that are likely to emerge in the presence/absence of a hierarchy by introducing the concept of two different learning methods - the vertical and horizontal learning methods.

Our study theorizes how organizations can achieve better learning performance in specific conditions, which previous studies have relatively unexplored and implicitly considered as background. We examined the three typical conditional factors of organizational learning - specifically problem complexity, member regrouping, and group size-and provided a hybrid for higher learning performance even when organizations cannot change their fundamental hierarchical structures. According to our study, these conditional factors can directly impact the performance of different organizations. High problem complexity may make it more difficult for hierarchical organizations to catch up to the learning performance of non-hierarchical organizations. However, an important implication of our study is that hierarchical organizations can significantly improve their performance by reducing the level of problem complexity or periodically introducing member regrouping. To reduce problem complexity, organizations may fragment the given problems into multiple sub-unit problems or decrease the possible interdependence among their sub-components. Alternatively, hierarchical organizations can increase the frequency of member regrouping to reduce the time necessary to catch up to the learning performance of non-hierarchical organizations. The problem 
complexity, regardless of whether it is high or low, did not change the effect of member regrouping on learning performance. Overall, we propose that organizational learning may be a process contingent upon the different configurations of multiple learning conditions.

Although we replicated three conditional factors in our simulation models, we suggest that future researchers alter the conditional factors to extend our framework. For example, we showed that the size of the project groups does matter when dealing with very complex problems especially for the organization using the vertical learning method. This finding may provide a meaningful research opportunity that compares the learning mechanisms of small enterprises versus large corporations. Future studies can further integrate more realistic conditions of organizational learning, such as frequent employee turnover, selection criteria for group leaders, sudden changes in organizational routines, conflict among group members, leadership style, inter- and intra-firm competition, and environmental conditions. For instance, the possible competition between individuals, groups, and organizations may significantly change the learning dynamics in knowledge intensive industries (Hamel 1991). Other studies showed that market leaders do have unique knowledge seeking procedures compared to market followers even in the same industry and at the same geographical location (Park, Mezias, Lee and Han 2014). Lastly, future studies can also refine our findings by incorporating cost models as one of the key condition which can alter the organizational learning performance. For example, in horizontal learning, acquiring and sharing different kinds of specialized knowledge across units can incur significant costs (Postrel 2002). By taking the cost approach, researchers can provide more insight into organizational design issues such as identifying the optimal level of division of knowledge and optimal layers of hierarchy.

From a managerial point of view, depending on the learning method, our study implies that the effect of hierarchical structure may deliver different impact on organizational learning, especially in various contexts: level of knowledge complexity, frequency of member regrouping, and the size of the project group. This is a strategically crucial factor in the high-tech sectors where the efficiency of sharing and integrating specialized knowledge directly affects corporate competitive advantage (Grant 1996). For successful outcomes, organizations using the vertical learning method may 
periodically reshuffle group members or rotate job assignments especially when they deal with complex tasks. Alternatively, hightech firms can reshape their learning mechanisms by adopting horizontal learning with a non-hierarchical structure.

Many organizations have recently achieved unprecedented favorable outcomes by adopting project-based temporal work groups within and across organizations (Hobday 2000). For example, W. L. Gore \& Associates, a high-tech manufacturing company known for its waterproof and breathable Gore-Tex fabrics, is also renowned for managing its organization with the absence of hierarchy. Without formal titles and even a formal group leader, Gore \& Associates created an excellent learning environment where employees willingly learn from each other, commit to open communications, and ultimately create breakthrough ideas. Our study advises that managers may need to pay special attention to their organizational structures and internal learning methods.

\section{REFERENCES}

Aoki, M. (1986), "Horizontal vs. vertical information structure of the firm," American Economic Review, 76(5), 971-983.

Arrow, H., and J. E. McGrath (1995), "Membership dynamics in groups at work: A theoretical framework," Research in Organizational Behavior, 17, 373-373.

Arrow, K. J. (1985), "Informational structure of the firm," American Economic Review, 75(2), 303-307.

Baldwin, C. Y., and K. B. Clark (2000), Design rules: The power of modularity. MIT press, Cambridge, MA.

Brady, T., and A. Davies (2004), "Building project capabilities: from exploratory to exploitative learning," Organization Studies, 25(9), 16011621.

Bunderson, J. S., and P. Boumgarden (2010), "Structure and learning in self-managed teams: Why "bureaucratic" teams can be better learners," Organization Science, 21(3), 609-624.

Burns, T. E., and G. M. Stalker (1961), The management of innovation. Tavistock, London.

Cannon-Bowers, J. A., and E. Salas (2001), "Reflections on shared cognition," Journal of Organizational Behavior, 22(2), 195-202.

Carley, K. (1992), "Organizational learning and personnel turnover," Organization Science, 3(1), 20-46. 
Castore, C. H., K. Peterson, and T. A. Goodrich (1971), "Risky shift: Social value or social choice? An alternative model," Journal of Personality and Social Psychology, 20(3), 487-494.

Choi, H.-S., and L. Thompson (2005), "Old wine in a new bottle: Impact of membership change on group creativity," Organizational Behavior and Human Decision Processes, 98(2), 121-132.

Cohen, W. M., and D. A. Levinthal (1990), "Absorptive capacity: a new perspective on learning and innovation," Administrative Science Quarterly, 35(1), 128-152.

Coleman, J. S. (1988), "Social capital in the creation of human capital," American Journal of Sociology, 94, 95-120.

Crossan, M. M., H. W. Lane, and R. E. White (1999), "An organizational learning framework: From intuition to institution," Academy of Management Review, 24(3), 522-537.

Daft, R. L., and R. H. Lengel (1986), "Organizational information requirements, media richness and structural design," Management Science, 32(5), 554-571.

Daft, R. L., and K. E. Weick (1984), "Toward a model of organizations as interpretation systems," Academy of Management Review, 9(2), 284295.

Darr, E. D., and T. R. Kurtzberg (2000), "An investigation of partner similarity dimensions on knowledge transfer," Organizational Behavior and Human Decision Processes, 82(1), 28-44.

Davis, J. H., N. L. Kerr, R. S. Atkin, R. Holt, and D. Meek (1975), "The decision processes of 6-and 12-person mock juries assigned unanimous and two-thirds majority rules," Journal of Personality and Social Psychology, 32(1), 1-14.

Edmondson, A. C., R. M. Bohmer, and G. P. Pisano (2001), "Disrupted routines: Team learning and new technology implementation in hospitals," Administrative Science Quarterly, 46(4), 685-716.

Fang, C., J. Lee, and M. A. Schilling (2010), "Balancing exploration and exploitation through structural design: The isolation of subgroups and organizational learning," Organization Science, 21(3), 625-642.

Fiol, C. M., and M. A. Lyles (1985), "Organizational learning," Academy of Management Review, 10(4), 803-813.

Foss, N. J. (2007), "The emerging knowledge governance approach: Challenges and characteristics," Organization, 14(1), 29-52.

Galbraith, J. R. (1974), "Organization design: An information processing view," Interfaces, 4(3), 28-36.

Gavetti, G. (2005), "Cognition and hierarchy: Rethinking the microfoundations of capabilities' development," Organization Science, 16(6), 599-617.

Gittelman, M. (2007), "Does geography matter for science-based firms? 
Epistemic communities and the geography of research and patenting in biotechnology," Organization Science, 18(4), 724-741.

Grant, R. M. (1996), "Toward a knowledge-based theory of the firm," Strategic Management Journal, 17(S2), 109-122.

Gruenfeld, D. H., P. V. Martorana, and E. T. Fan (2000), "What do groups learn from their worldliest members? Direct and indirect influence in dynamic teams," Organizational Behavior and Human Decision Processes, 82(1), 45-59.

Gupta, A. K., P. E. Tesluk, and M. S. Taylor (2007), "Innovation at and across multiple levels of analysis," Organization Science, 18(6), 885-897.

Hamel, G. (1991), "Competition for competence and inter-partner learning within international strategic alliances," Strategic Management Journal, 12(4), 83-103.

Hansen, M. T. (1999), "The search-transfer problem: The role of weak ties in sharing knowledge across organization subunits," Administrative Science Quarterly, 44(1), 82-111.

Hansen, M. T. (2002), "Knowledge networks: Explaining effective knowledge sharing in multiunit companies," Organization Science, 13(3), 232-248.

Hayward, M. L. (2002), "When do firms learn from their acquisition experience? Evidence from 1990-1995," Strategic Management Journal, 23(1), 21-39.

Hobday, M. (2000), "The project-based organisation: an ideal form for managing complex products and systems?," Research Policy, 29(7), 871-893.

Huber, G. P. (1991), "Organizational learning: The contributing processes and the literatures," Organization Science, 2(1), 88-115.

Jablin, F. M., L. L. Putnam, K. H. Roberts, and L. W. Porter (1987), Handbook of organizational communication: An interdisciplinary perspective. Sage Newbury Park, CA.

Jansen, J. J., F. A. Van Den Bosch, and H. W. Volberda (2005), "Managing potential and realized absorptive capacity: how do organizational antecedents matter?" Academy of Management Journal, 48(6), 9991015.

Kane, A. A., L. Argote, and J. M. Levine (2005), "Knowledge transfer between groups via personnel rotation: Effects of social identity and knowledge quality," Organizational Behavior and Human Decision Processes, 96(1), 56-71.

Kauffman, S. (1995), At home in the universe: The search for the laws of selforganization and complexity. Oxford University Press, New York.

Kim, T., and M. Rhee (2009), "Exploration and exploitation: Internal variety and environmental dynamism," Strategic Organization, 7(1), 11-41.

Knudsen, T., and D. A. Levinthal (2007), "Two faces of search: Alternative generation and alternative evaluation," Organization Science, 18(1), 39- 
54.

Kogut, B., and U. Zander (1992), "Knowledge of the firm, combinative capabilities, and the replication of technology," Organization Science, 3(3), 383-397.

Lawson, C., and E. Lorenz (1999), "Collective learning, tacit knowledge and regional innovative capacity," Regional Studies, 33(4), 305-317.

Levine, J. M., and R. L. Moreland (1994), "Group socialization: Theory and research," European Review of Social Psychology, 5(1), 305-336.

Levinthal, D. A., and J. G. March (1993), "The myopia of learning," Strategic Management Journal, 14(S2), 95-112.

Levitt, B., and J. G. March (1988), "Organizational learning," Annual Review of Sociology, 14, 319-340.

Majchrzak, A., L. P. Cooper, and O. E. Neece (2004), "Knowledge reuse for innovation," Management Science, 50(2), 174-188.

March, J. G. (1991), "Exploration and exploitation in organizational learning," Organization Science, 2(1), 71-87.

March, J. G. (1994), Primer on decision making: How decisions happen. Free Press, New York.

March, J. G., and J. P. Olsen (1975), "The uncertainty of the past: Organizational learning under ambiguity," European Journal of Political Research, 3(2), 147-171.

Mihm, J., C. H. Loch, D. Wilkinson, and B. A. Huberman (2010), "Hierarchical structure and search in complex organizations," Management Science, 56(5), 831-848.

Miller, K. D., M. Zhao, and R. J. Calantone (2006), "Adding interpersonal learning and tacit knowledge to March's exploration-exploitation model," Academy of Management Journal, 49(4), 709-722.

Moorman, C., and A. S. Miner (1997), "The impact of organizational memory on new product performance and creativity," Journal of Marketing Research, 34(1), 91-106.

Moreland, R. L., and J. M. Levine (1982), "Socialization in small groups: Temporal changes in individual-group relations," Advances in Experimental Social Psychology, 15, 137-192.

Mueller, F. (1994), "Teams between hierarchy and commitment: Change strategies and the internal environment," Journal of Management Studies, 31(3), 383-403.

Naylor, J. C., and G. E. Briggs (1965), "Team-training effectiveness under various conditions," Journal of Applied Psychology, 49(4), 223.

Nickerson, J. A., and T. R. Zenger (2004), "A knowledge-based theory of the firm-The problem-solving perspective," Organization Science, 15(6), 617-632.

Nonaka, I. (1994), "A dynamic theory of organizational knowledge creation," Organization Science, 5(1), 14-37. 
Nonaka, I., and G. Von Krogh (2009), "Perspective-tacit knowledge and knowledge conversion: Controversy and advancement in organizational knowledge creation theory," Organization Science, 20(3), 635-652.

Park, N. K., J. M. Mezias, J. Lee, and J.-H. Han (2014), "Reverse knowledge diffusion: Competitive dynamics and the knowledge seeking behavior of Korean high-tech firms," Asia Pacific Journal of Management, 31(2), 355-375.

Pentland, B. T., and H. H. Rueter (1994), "Organizational routines as grammars of action," Administrative Science Quarterly, 484-510.

Perretti, F., and G. Negro (2006), "Filling empty seats: How status and organizational hierarchies affect exploration versus exploitation in team design," Academy of Management Journal, 49(4), 759-777.

Pisano, G. P., R. M. Bohmer, and A. C. Edmondson (2001), "Organizational differences in rates of learning: Evidence from the adoption of minimally invasive cardiac surgery," Management Science, 47(6), 752768.

Postrel, S. (2002), "Islands of shared knowledge: Specialization and mutual understanding in problem-solving teams," Organization Science, 13(3), 303-320.

Radner, R. (1993), "The organization of decentralized information processing," Econometrica: Journal of the Econometric Society, 61(5), 1109-1146.

Rivkin, J. W., and N. Siggelkow (2003), "Balancing search and stability: Interdependencies among elements of organizational design," Management Science, 49(3), 290-311.

Rosenkopf, L., and P. Almeida (2003), "Overcoming local search through alliances and mobility," Management Science, 49(6), 751-766.

Schulz, M. (2001), "The uncertain relevance of newness: Organizational learning and knowledge flows," Academy of Management Journal, 44(4), 661-681.

Senge, P. M. (1990), The fifth discipline: The art and practice of the learning organization. Doubleday, New York.

Siggelkow, N., and D. A. Levinthal (2005), "Escaping real (non-benign) competency traps: Linking the dynamics of organizational structure to the dynamics of search," Strategic Organization, 3(1), 85-115.

Siggelkow, N., and J. W. Rivkin (2005), "Speed and search: Designing organizations for turbulence and complexity," Organization Science, 16(2), 101-122.

Simon, H. A., and P. A. Simon (1962), "Trial and error search in solving difficult problems: Evidence from the game of chess," Behavioral Science, 7(4), 425-429.

Singley, M. K., and J. R. Anderson (1989), The transfer of cognitive skill. Harvard University Press, Cambridge, MA.

Szulanski, G. (1996), "Exploring internal stickiness: Impediments to the 
transfer of best practice within the firm," Strategic Management Journal, 17(S2), 27-43.

Tsai, W. (2001), "Knowledge transfer in intraorganizational networks: Effects of network position and absorptive capacity on business unit innovation and performance," Academy of Management Journal, 44(5), 996-1004.

Weber, R. A., and C. F. Camerer (2003), "Cultural conflict and merger failure: An experimental approach," Management Science, 49(4), 400415.

Williamson, O. E. (1991), "Comparative economic organization: The analysis of discrete structural alternatives," Administrative Science Quarterly, 36(2), 269-296.

Zander, U., and B. Kogut (1995), "Knowledge and the speed of the transfer and imitation of organizational capabilities: An empirical test," Organization Science, 6(1), 76-92.

Zollo, M., and S. G. Winter (2002), "Deliberate learning and the evolution of dynamic capabilities," Organization Science, 13(3), 339-351.

Received September 10, 2015

Revision Received November 23, 2015

Accepted November 23, 2015 